\title{
Evaluation of Symptom and Side Effect Bother in Cutaneous T-cell Lymphoma Patients Treated with Mogamulizumab or Vorinostat
}

Stacie Hudgens ( $\nabla$ stacie.hudgens@clinoutsolutions.com )

Clinical Outcomes Solutions https://orcid.org/0000-0002-7350-1549

Pierluigi Porcu

Thomas Jefferson University

Pietro Quaglino

University of Turin: Universita degli Studi di Torino

Auris Huen

MD Anderson Cancer Center

Lysbeth Floden

Clinical Outcomes Solutions

\section{Karen Dwyer}

Kyowa Kirin Pharmaceutical Development, Ind

Madeleine Duvic

MD Anderson Cancer Center

\section{Research}

Keywords: cutaneous T-cell lymphoma, CTCL, item analysis, Skindex-29, FACT-G, mogamulizumab, vorinostat, health related quality of life

Posted Date: June 8th, 2021

DOI: https://doi.org/10.21203/rs.3.rs-566715/v1

License: (a) (1) This work is licensed under a Creative Commons Attribution 4.0 International License. Read Full License

Version of Record: A version of this preprint was published at Blood on November 29th, 2018. See the published version at https://doi.org/10.1182/blood-2018-99-116346. 


\section{Abstract}

Background: Cutaneous T-cell lymphomas, including mycosis fungoides or Sézary syndrome, are rare non-Hodgkin lymphomas that can be serious and life-threatening with significant morbidity and impaired quality of life. The purpose of this study was to determine whether patient-reported outcome individual items on skin symptoms and toxicity bother show significant treatment differences for patients with mycosis fungoides or Sézary syndrome.

Methods: Health-related quality of life was assessed during the MAVORIC clinical trial of mogamulizumab for treatment of cutaneous T-cell lymphomas compared to vorinostat using the Skindex-29 (measures emotions, symptoms, and functioning) and Functional Assessment of Cancer Therapy - General (FACT-G) (assesses physical, social/family, emotional, and functional well-being). Pruritus was assessed with the ItchyQoL (measures symptoms, functioning, and emotions) and Likert Scale for level of itching. Item analyses used longitudinal generalized estimation equations of the postbaseline, treated period assessments to estimate likelihood of improvement. Forest plots of odds ratios (OR) characterized the likelihood of a 1-and 2-grade categorical improvement on individual items.

Results: Patients treated with mogamulizumab were more likely to observe a 1-grade improvement in painful skin (OR $=1.74, \mathrm{Cl}: 1.180-2.572)$, irritated skin $(\mathrm{OR}=1.34, \mathrm{Cl}: 0.909-1.978)$, lack of energy $(\mathrm{OR}=$ 2.20 , Cl: $1.461-3.309)$, side effect bother $(\mathrm{OR}=1.28, \mathrm{Cl}: 0.810-2.020)$, and general cancer pain $(\mathrm{OR}=$ 1.38, Cl: 0.922-2.063). Patients treated with mogamulizumab were more likely to observe a 2-grade improvement in burning or stinging skin $(\mathrm{OR}=1.62, \mathrm{Cl}: 1.013-2.591, \mathrm{P}<0.05)$ and more likely to observe improvement in painful skin $(\mathrm{OR}=1.78, \mathrm{Cl}: 1.045-3.021, \mathrm{P}<0.05)$.

Conclusions: Single item analyses suggest a mogamulizumab symptom benefit during treatment compared to vorinostat for items on the skin symptoms of Skindex-29 and toxicity bother items of FACTG.

\section{Trial registration: NCT01728805}

\section{Background}

Cutaneous T-cell lymphomas (CTCLs) represent a rare form of non-Hodgkin lymphomas that primarily affects the skin, presenting as patches, plaques, tumors or erythroderma, and may be associated with severe pruritus $[1,2]$. The two most common subtypes, accounting for approximately $50 \%$ of all CTCL, are mycosis fungoides (MF) and Sézary syndrome (SS) [3]. MF is a mature T-cell non-Hodgkin lymphoma with presentation in the skin and potential involvement of the nodes, blood, and viscera. SS is defined as an erythrodermic CTCL with a leukemic involvement of malignant T-cells clonally matching that in the skin. In contrast to patch/plaque MF, SS is much more symptomatic, has a lower potential for remission, and lower expected survival. Advanced CTCL can be a serious, life-threatening disease associated with significant morbidity and impaired health-quality of life (HRQoL) [4-6]. 
MAVORIC was the first pivotal trial in CTCL to use progression free survival (PFS) as a primary endpoint. It was also the largest randomized study to compare systemic therapies in MF and SS subtypes of CTCL. MAVORIC evaluated the efficacy of the anti-CC chemokine receptor type 4 (CCR4)-targeted antibody, mogamulizumab, for the treatment of MF or SS compared to that of vorinostat (NCT01728805) [7]. Mogamulizumab had significantly better PFS compared to vorinostat (median PFS of 7.7 months for mogamulizumab and 3.1 months for vorinostat $[\mathrm{P}<0.0001])$. Global overall response rate was significantly improved in the patients randomized to mogamulizumab at $28.0 \%$ versus $4.8 \%$ for vorinostat $(P<0.0001)$. The most common treatment-emergent adverse events (TEAEs) in the mogamulizumab arm included infusion-related reactions (33.2\% versus $0.5 \%$ in the vorinostat arm) and drug rash $(23.9 \%$ versus $0.5 \%)$. Common TEAEs reported more often with vorinostat included diarrhea ( $61.8 \%$ versus $23.4 \%$ in the mogamulizumab arm), nausea ( $42.5 \%$ versus $15.2 \%$ ), thrombocytopenia (30.6\% versus $11.4 \%$ ), dysgeusia (29.0\% versus $3.3 \%$ ), and increased blood creatinine $(28.0 \%$ versus $3.3 \%$ ). Preplanned analyses of patient reported outcomes found mogamulizumab had a greater improvement in HRQoL measures at the 6-month assessment than did vorinostat treated patients $(P>$ 0.05) [7].

The objective of this analysis was to determine whether individual items related with skin symptoms and safety profile on patient-reported outcome measures show significant differences over the course of treatment for patients with CTCL.

\section{Methods}

Details of the study design have been published (NCT01728805) [7]. Briefly, this was a large, open-label, multi-center, randomized, Phase 3 study that compared mogamulizumab versus vorinostat in 372 patients who had failed $\geq 1$ prior systemic therapy for MF or SS (Figure 1). Eligible patients were at least 18 years of age, had an Eastern Cooperative Oncology Group performance score of $\leq 1$, and had adequate hematologic, hepatic, and renal function. Patients were randomly assigned (1:1) to mogamulizumab $(1.0 \mathrm{mg} / \mathrm{kg}$ intravenously weekly for 4 weeks of the first 28-day cycle, then on Days 1 and 15 of subsequent cycles) or vorinostat ( $400 \mathrm{mg}$ daily). Stratification was by cutaneous T-cell lymphoma subtype (MF versus SS) and disease stage (IB-II versus III-IV).

\section{Study Instruments}

Health-related quality of life was assessed using the Skindex-29 and Functional Assessment of Cancer Therapy - General (FACT-G). Evaluation of pruritus was assessed using the ItchyQoL and the Pruritus Likert scale. The Skindex-29 and FACT-G were evaluated at Baseline (Cycle 1, Day 1), end of Cycle 1, then every 8 weeks (ie, end of Cycle 3, 5, .., 25) through Cycle 25, and at the End of Treatment visit. The ItchyQoL and the Pruritus Likert scale were evaluated every 4 weeks at each cycle. Measurements at the end of Cycle 1 were defined as the latest value obtained from Cycle 1 (Days 26 through 28) or Cycle 2 (Day 1). Measurements at the end of Cycle $X$, where $x=1,2,3, \ldots, 25$ were defined as the latest value 
obtained from Cycle X (Days 26 through 28), Cycle X every 8 weeks, or Cycle $(X+1)$ Day 1. Clinical HRQoL measurements included Skindex-29, FACT-G, and two measures of pruritus (a Likert Scale and ItchyQoL; not included in this presentation).

The Skindex-29 is a validated instrument to measure the effect of skin disease on HRQoL [8]. It is composed of 29 items assessing three domains: emotions, symptoms, and functioning. The items are scored on a 5-point Likert-type scale (never, rarely, sometimes, often, or all the time). Responses to each item are transformed to a linear scale of 100 (where $0=$ never, $25=$ rarely, $50=$ sometimes, $75=$ often, and $100=$ all the time) for the purpose of scale score calculation. A scale score is the mean of a subject's responses to the items in a given scale and the composite Skindex-29 score is calculated as the average of the three scale scores to measure the overall impact on HRQoL. Higher scores indicate a higher impact of skin disease on emotions, symptoms, and functioning.

The FACT-G questionnaire is used to assess effects of underlying disease and its treatment on patient HRQoL [9]. As a generic cancer-related core, the FACT-G includes the 27-item FACT-General (FACT-G) to assess symptoms and treatment related effects impacting physical well-being (seven items), social/family well-being (seven items), emotional well-being (six items), and functional well-being (seven items). Scores for the domain subscales can be combined to produce a FACT-G total score, which provides an overall indicant of generic HRQoL. The recall period is 1 week. Each question is assessed on a 5-point Likert scale: $0=$ not at all, $1=$ a little bit, $2=$ somewhat, $3=$ quite a bit, and $4=$ very much. The FACT-G scoring guide identifies those items that must be reversed before being added to obtain subscale totals. Negatively stated items are reversed by subtracting the response from "4." After reversing proper items, all subscale items are summed to a total, which is the subscale score. For scales and symptom indices, higher scores represent better HRQoL.

\section{Statistical Analysis}

The analytic population was defined as patients with clinical outcome assessments at any time point within the intent-to-treat population. These exploratory post-hoc analyses were performed using the Statistical Analysis System Version 9.4.

Analyses on identified individual symptom items of Skindex-29 and toxicity items of FACT-G were conducted using descriptive, categorical change from baseline as well as longitudinal generalized estimation equations (GEE) of the post-baseline, treated period assessments (through Cycles 5 or 6 , depending on collection schedule) for these items. Generalized estimation equations of logistic regression models were used to estimate the likelihood of improvement. The GEE model of an "improved" binary event was regressed on fixed effects of treatment arm, time point, and their interaction terms, as well as an R-side random effect with autoregressive correlation structure to account for repeated measures. Model results were used to generate the odds ratios and the $95 \%$ confidence interval to compare the likelihood of "improvement" between treatment groups. The proportion of patients 
experiencing a 1-grade and 2-grade improvement is presented in terms of frequency and percentage by treatment arm.

Forest plots of odds ratios (OR) and associated confidence intervals $(\mathrm{Cl})$ from the GEE analyses were generated to characterize the likelihood of a 1-grade (eg, improvement by 1 category on the verbal response scale) and 2-grade (eg, improvement by two categories on the verbal response scale) categorical improvement on individual items for patients treated with mogamulizumab compared to vorinostat during the first 6 cycles of therapy.

\section{Results}

Treatment groups were balanced with respect to demographic and physical characteristics, disease characteristics, and previous therapies for MF/SS without statistically significant differences (Table 1).

\section{Table 1}

\section{Baseline Characteristics}




\begin{tabular}{|c|c|c|c|}
\hline Demographic or Clinical Item & $\begin{array}{l}\text { Mogamulizumab } \\
(n=186)\end{array}$ & $\begin{array}{l}\text { Vorinostat } \\
(n=186)\end{array}$ & $\begin{array}{l}\text { Total } \\
(\mathrm{N}=372)\end{array}$ \\
\hline \multicolumn{4}{|l|}{ Age group } \\
\hline$<65$ & $99(53.2 \%)$ & $89(47.8 \%)$ & $188(50.5 \%)$ \\
\hline$\geq 65$ & $87(46.8 \%)$ & $97(52.2 \%)$ & $184(49.5 \%)$ \\
\hline \multicolumn{4}{|l|}{ Gender } \\
\hline Female & $77(41.4 \%)$ & 79 (42.5\%) & $156(41.9 \%)$ \\
\hline Male & $109(58.6 \%)$ & $107(57.5 \%)$ & $216(58.1 \%)$ \\
\hline \multicolumn{4}{|l|}{ Race } \\
\hline Black/African American & $24(12.9 \%)$ & $13(7.0 \%)$ & $37(9.9 \%)$ \\
\hline White & $125(67.2 \%)$ & $135(72.6 \%)$ & $260(69.9 \%)$ \\
\hline Other & $37(19.9 \%)$ & $38(20.4 \%)$ & $75(20.2 \%)$ \\
\hline \multicolumn{4}{|l|}{ Disease type } \\
\hline MF & $105(56.5 \%)$ & 99 (53.2\%) & $204(54.8 \%)$ \\
\hline SS & $81(43.5 \%)$ & $87(46.8 \%)$ & $168(45.2 \%)$ \\
\hline \multicolumn{4}{|l|}{ Disease stage } \\
\hline IB or II & $68(36.6 \%)$ & $72(38.7 \%)$ & $140(37.6 \%)$ \\
\hline III or IV & $118(63.4 \%)$ & $114(61.3 \%)$ & $232(62.4 \%)$ \\
\hline \multicolumn{4}{|l|}{ Blood involvement } \\
\hline Yes & $123(66.1 \%)$ & $122(66.3 \%)$ & $245(66.2 \%)$ \\
\hline No & $63(33.9 \%)$ & $62(33.7 \%)$ & $125(33.8 \%)$ \\
\hline Missing/no response ${ }^{a}$ & 0 & 2 & 2 \\
\hline \multicolumn{4}{|l|}{ Region } \\
\hline US & $98(52.7 \%)$ & $103(55.4 \%)$ & $201(54.0 \%)$ \\
\hline Japan & $9(4.8 \%)$ & $6(3.2 \%)$ & $15(4.0 \%)$ \\
\hline Rest of World ${ }^{b}$ & 79 (42.5\%) & 77 (41.4\%) & $156(41.9 \%)$ \\
\hline
\end{tabular}




\begin{tabular}{|c|c|c|c|}
\hline Demographic or Clinical Item & $\begin{array}{l}\text { Mogamulizumab } \\
(n=186)\end{array}$ & $\begin{array}{l}\text { Vorinostat } \\
(\mathrm{n}=186)\end{array}$ & $\begin{array}{l}\text { Total } \\
(\mathrm{N}=372)\end{array}$ \\
\hline \multicolumn{4}{|c|}{$\begin{array}{l}\text { Abbreviations: MF = mycosis fungoides; SS = Sézary syndrome; US = United States } \\
\text { a Percent missing is overall by treatment group and are not included in the calculation of response } \\
\text { proportions. }\end{array}$} \\
\hline
\end{tabular}

Data demonstrated that subjects treated with mogamulizumab more often showed an improvement in or no worsening of their HRQoL versus those treated with vorinostat $(O R>1.0)$. Patients treated with mogamulizumab were more likely to observe a 1-grade improvement in painful skin $(\mathrm{OR}=1.74, \mathrm{Cl}$ : 1.180-2.572), irritated skin ( $O R=1.34, \mathrm{Cl}: 0.909-1.978)$, lack of energy $(\mathrm{OR}=2.20, \mathrm{Cl}: 1.461-3.309)$, side effect bother $(\mathrm{OR}=1.28, \mathrm{Cl}: 0.810-2.020)$, and general cancer pain $(\mathrm{OR}=1.38, \mathrm{Cl}: 0.922-2.063)$ within 6 cycles of therapy (Figure $2 A$ ). Patients treated with mogamulizumab were $62 \%$ more likely to observe a 2grade improvement in burning or stinging skin $(\mathrm{OR}=1.62, \mathrm{Cl}: 1.013-2.591, \mathrm{P}<0.05)$ and $78 \%$ more likely to observe this level of improvement in painful skin $(\mathrm{OR}=1.78, \mathrm{Cl}: 1.045-3.021, \mathrm{P}<0.05)$ within 6 cycles of therapy (Figure 2B). While not statistically significant, mogamulizumab patients were $60 \%$ more likely to report 2-grade improvement in side effect bother associated with treatment $(\mathrm{OR}=1.60, \mathrm{Cl}: 0.85-3.009$, $P<0.05)$ within 5 cycles of therapy.

The single item descriptive and proportion analysis of 1-grade and 2-grade improvement at Cycle 5 from baseline favors mogamulizumab compared with vorinostat (Table 2). Data demonstrate that subjects treated with mogamulizumab more often showed an improvement in or no worsening of their quality of life versus those subjects treated with vorinostat. For the 1-grade improvement, mogamulizumab-treated patients had significant improvements versus vorinostat for the items: skin hurts $(P=0.02)$; bothered by side effects $(P=0.01)$; and lack of energy $(P=0.05)$. For the 2-grade improvement, mogamulizumabtreated patients had significant improvements versus vorinostat for the items: skin hurts $(P<0.05)$ and pain $(P=0.02)$.

Table 2

Single Item Analysis of Improvement at Cycle 5 or 6 from Baseline 


\begin{tabular}{|llll|}
\hline Item Name & Mogamulizumab $^{\mathrm{a}}$ & Vorinostat $^{\mathrm{a}}$ & P-value \\
\hline 1-grade improvement & & & \\
\hline SKINDEX-29: My skin condition bleeds & $38(40.9 \%)$ & $20(37.7 \%)$ & 0.7106 \\
\hline SKINDEX-29: My skin is irritated & $56(59.6 \%)$ & $26(49.1 \%)$ & 0.2176 \\
\hline SKINDEX-29: My skin itches & $53(56.4 \%)$ & $30(56.6 \%)$ & 0.9793 \\
\hline SKINDEX-29: My skin condition burns or stings & $49(52.1 \%)$ & $27(50.9 \%)$ & 0.8903 \\
\hline SKINDEX-29: My skin hurts & $53(56.4 \%)$ & $19(36.5 \%)$ & 0.0216 \\
\hline FACT-G: I am bothered by side effects of treatment & $32(33.3 \%)$ & $7(14.0 \%)$ & 0.0122 \\
\hline FACT-G: I have pain & $43(43.4 \%)$ & $15(28.8 \%)$ & 0.0799 \\
\hline FACT-G: I have nausea & $10(10.1 \%)$ & $4(7.4 \%)$ & 0.7713 \\
\hline FACT-G: I have a lack of energy & $43(43.9 \%)$ & $15(27.8 \%)$ & 0.0505 \\
\hline 2-grade improvement & & & \\
\hline SKINDEX-29: My skin condition bleeds & $15(16.1 \%)$ & $3(5.7 \%)$ & 0.0721 \\
\hline SKINDEX-29: My skin is irritated & $32(34.0 \%)$ & $14(26.4 \%)$ & 0.3382 \\
\hline SKINDEX-29: My skin itches & $28(29.8 \%)$ & $11(20.8 \%)$ & 0.2336 \\
\hline SKINDEX-29: My skin condition burns or stings & $29(30.9 \%)$ & $14(26.4 \%)$ & 0.5703 \\
\hline SKINDEX-29: My skin hurts & $26(27.7 \%)$ & $7(13.5 \%)$ & 0.0495 \\
\hline FACT-G: I am bothered by side effects of treatment & $14(14.6 \%)$ & $2(4.0 \%)$ & 0.0562 \\
\hline FACT-G: I have pain & $22(22.2 \%)$ & $4(7.7 \%)$ & 0.0249 \\
\hline FACT-G: I have nausea & $3(3.0 \%)$ & $1(1.9 \%)$ & 1.0000 \\
\hline FACT-G: I have a lack of energy & $11(11.2 \%)$ & $4(7.4 \%)$ & 0.5754 \\
\hline a Percentages are based on those who adhered or remained in the study & up until Cycle 5 or 6. \\
\hline
\end{tabular}

\section{Discussion}

Patient reported outcome measures included in this study were multi-item scales that represent uni- or multi-dimensional concepts related to symptomatic manifestation, cancer-related quality of life, and tolerability. Interpretation of the individual items further supports the overall interpretability of the domain-level scores on the respective domains. More specifically, while the Skindex items represent the 
core skin symptom manifestations (eg, bleeding, irritation, itching, burning, or hurting), the individual symptoms may respond to treatment differently and should be assessed individually.

Understanding the specific items that are contributing most to the total score is important in evaluating treatment efficacy as the individual items represent cardinal components of HRQoL. By isolating each item in a categorical analysis and setting a threshold for meaningful categorical change, which in this analysis was 1-grade change threshold on the respective 5-point Skindex and 4-point FACT-G verbal response scales, analogous to $20 \%$ and $25 \%$ categorical improvement, with sensitivity testing using a more conservation 2 -grade change ( $40 \%$ and $50 \%$ categorical improvement), it is possible to determine how the overall symptoms move on the total score, thus enhancing the interpretability $[10,11]$.

In a previous study, mogamulizumab was found to gain a greater improvement in HRQoL measures at the 6-month assessment than did vorinostat [7]. In the present analysis, we confirm that mogamulizumab was able to induce a significant greater improvement on individual items related with skin symptoms and safety profile in the same patient cohort.

These results provide a detailed account of the magnitude at which HRQoL was affected by treatment with mogamulizumab.

A limitation of the analysis is a lack of data in the later cycles of treatment, such as post-progression, as well as reasons for missing PRO data. Further data on durability of symptomatic and functional improvements in HRQoL and influence of progression on these patient outcomes could prove valuable for considering the long-term ramifications of treatment selection. Future prospective studies using singleitem analyses should focus on long-term changes in HRQoL.

\section{Conclusion}

Single item analyses provide detailed information on the cumulative probability of categorical improvement of individual items on the skin symptoms of Skindex-29 and the toxicity bother items of FACT-G. These results suggest a symptom benefit of mogamulizumab over the course of treatment compared to vorinostat.

\section{Abbreviations}

$\mathrm{Cl}$ - confidence interval; CTCL, cutaneous T-cell lymphoma; HRQoL - health related quality of life; LCL lower confidence limit; MF - mycosis fungoides; OR - odds ratio; PFS - progression free survival; SS Sézary syndrome; TEAEs - treatment-emergent adverse events; UCL - upper confidence limit; US United States

\section{Declarations}


All study procedures were in accordance with ethical standards of the 1964 Helsinki Declaration and its later amendments, relevant laws, institutional guidelines, and approved by an institutional review board (IRB). The protocol was reviewed and approved by institutional review boards or independent ethics committees at each study center.

\section{Participant Consent for Publication}

Not applicable

\section{Availability of Data and Materials}

The datasets generated and/or analyzed during the current study are not publicly available, but are available from the corresponding author on reasonable request.

\section{Competing Interests}

$\mathrm{KD}$ is an employee of Kyowa Kirin Pharmaceutical, Inc. SH, PP, PQ, AH, LF, and MD declare no competing interests.

\section{Funding}

This study was funded by Kyowa Kirin Pharmaceutical, Inc.

\section{Authors' contributions}

All authors contributed to the conception, analysis, interpretation, and revised manuscript content. All authors read and approved the final manuscript.

\section{Acknowledgements:}

Editorial services were provided by Chad Green at Clinical Outcomes Solutions.

\section{References}

1. Horwitz S, Olsen E, Duvic M, Porcu P, Kim Y. Review of the treatment of mycosis fungoides and Sézary syndrome: a stage-based approach. J Natl Compr Canc Netw. 2008;6(4):436-442.

2. Meyer N, Paul C, Misery L. Pruritus in cutaneous T-cell lymphomas: frequent, often severe and difficult to treat. Acta Derm Venereol. 2010;90(1):12-17. 
3. Willemze R, Jaffe E, Burg G, et al. WHO-EORTC classification for cutaneous lymphomas. Blood. 2005;105(10):3768-3785.

4. demierre $\mathrm{M}, \mathrm{Whittaker} \mathrm{S}$, Kim $\mathrm{Y}$, et al. Pooled analyses of two international, multicenter clinical studies of romidepsin in 167 patients with cutaneous T-cell lymphoma (CTCL). J Clin Oncol. 2009;27(15 Suppl):8546.

5. Demierre M, Tien A, Miller D. Health-related quality-of-life assessment in patients with cutaneous Tcell lymphoma. Arch Dermatol. 2005;141(3):325-330.

6. Demierre M, Gan S, Jones J, Miller D. Significant impact of cutaneous T-cell lymphoma on patients' quality of life. Cancer. 2006;107(10):2504-2511.

7. Kim Y, Bagot M, Pinter-Brown L, et al. Mogamulizumab versus vorinostat in previously treated cutaneous T-cell lymphoma (MAVOIRC): an international, open-label, randomised, controlled phase 3 trial. Lancet Oncol. 2018;19(9):1192-1204.

8. Chren M, Lasek R, Flocke S, Zyzanski S. Improved discriminative and evaluative capability of a refined version of Skindex, a quality-of-life instrument for patients with skin diseases. Arch Dermatol. 1997;133:1433-1440.

9. Cella D, Tulsky D, Gray G, et al. The Functional Assessment of Cancer Therapy scale: development and validation of the general measure. J Clin Oncol. 1993;11(3):570-579.

10. Hudgens S, Floden L, Symonds T, Campbell A, Slagle A. Interpreting disease symptoms: item-level analysis methodology. Qual Life Res. 2019;28:S165-S166.

11. Hudgens S, Floden L, Symonds T, Slagle A. PRM9-A Longitudinal, item-level analysis to support the interpretability of multi-item patient reported outcomes. Value Health. 2018;21(Suppl 3):S357.

\section{Figures}

\section{Inclusion:}

-Stage IB - IVB

histologically

confirmed MF or SS

-At least one prior course of systemic therapy

Exclusion:

-Patients with large cell transformation

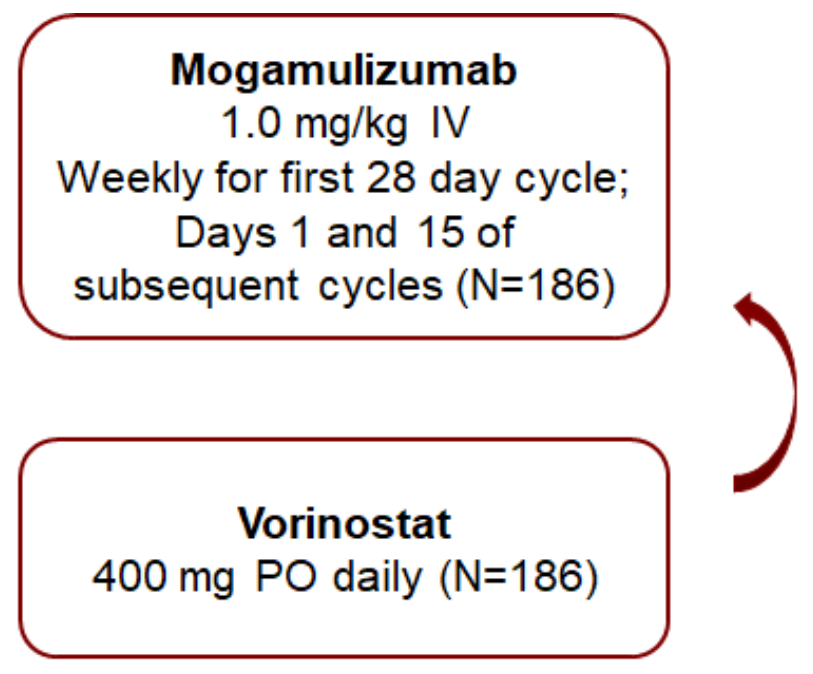

One-way crossover after PD or intolerable toxicity

\section{Figure 1}


MAVORIC Study Design Abbreviations: IV = intravenous; MF = mycosis fungoides; PD = progression of disease; PO = orally; SS = Sézary syndrome; US = United States

\section{A. 1-Grade Improvement}

\section{SkinDex-29}

Skin condition bleeds

Skin is irritated

Skin itches

Skin burns or stings

Skin hurts

\section{FACT-G}

Treatment side effects bother

Pain

Nausea

Lack of energy

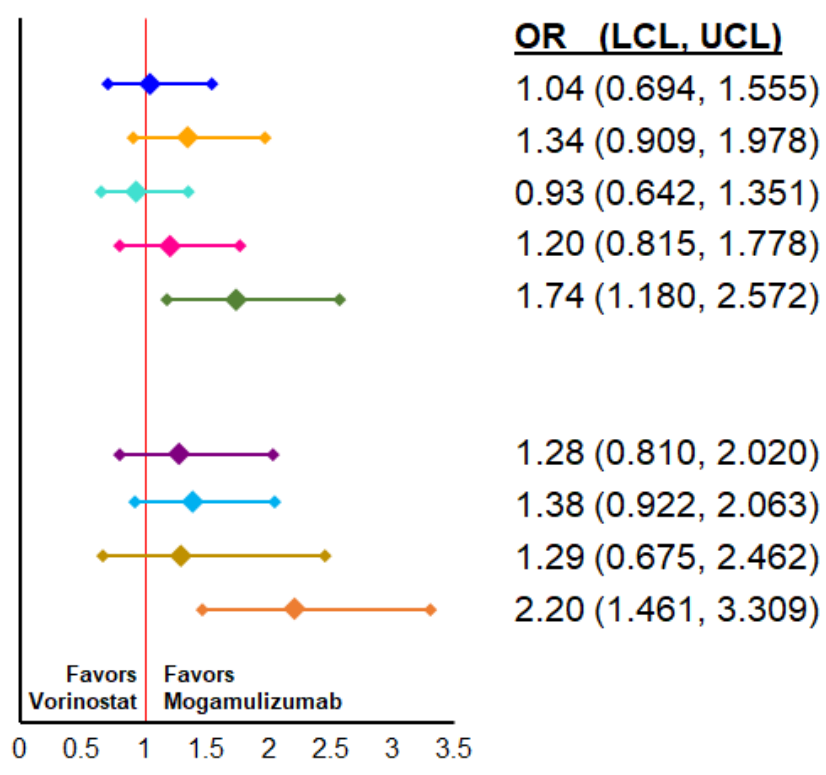

Odds Ratio

\section{B. 2-Grade Improvement}

\section{Skindex-29}

Skin condition bleeds

Skin is irritated

Skin itches

Skin burns or stings

Skin hurts

\section{FACT-G}

Treatment side effects bother

Pain

Nausea

Lack of energy

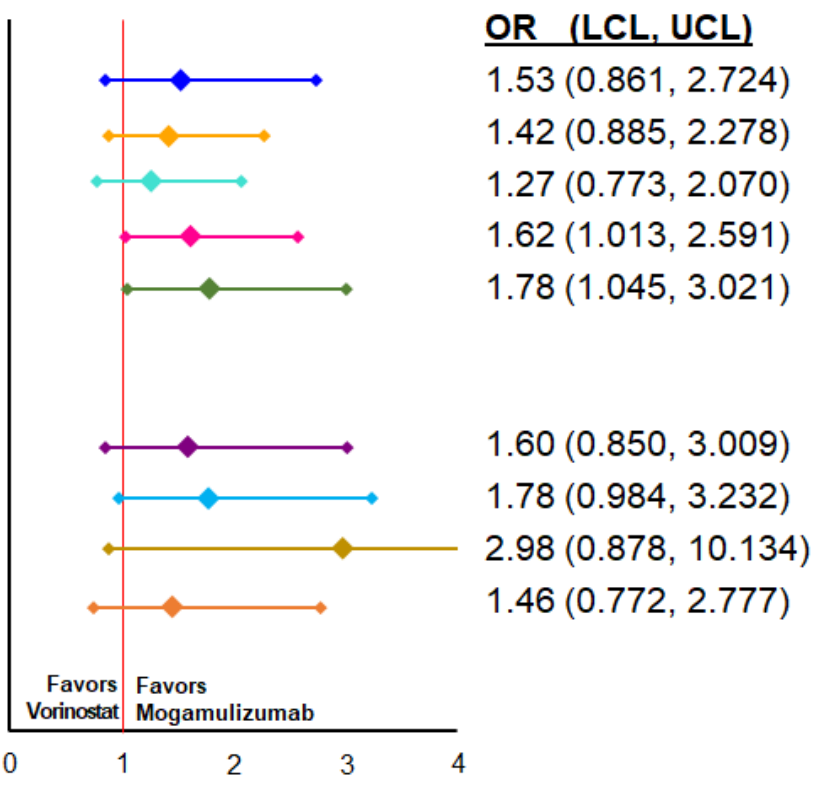

Odds Ratio

\section{Figure 2}

Probability of Observing Categorical Improvement on Individual Items Through Cycle 5 or 6 Abbreviations: FACT-G = Functional Assessment of Cancer Therapy - General; $L C L=$ lower confidence limit; $\mathrm{OR}$ = odds ratio; $\mathrm{UCL}=$ upper confidence limit 